\title{
Gewalt und emotionale Energie
}

\author{
Thomas Kron
}

(C) Der/die Autor(en) 2020

Zusammenfassung Der vorliegende Beitrag entwickelt Randall Collins' Theorie situationalistischer Gewalthandlungen in dem zentralen Aspekt der emotionalen Energie weiter. Erstens ergänze ich Collins' Vorstellung positiver emotionale Energie um den Faktor negativer emotionaler Energie. Zweitens modelliere ich diese beiden Energieformen als Mengen und beziehe diese dynamisch aufeinander. Damit kommt der Entropie emotionaler Energie als neuer Faktor der Gewaltentstehung eine Schlüsselrolle zu, die bislang vernachlässigt wurde.

Schlüsselwörter Gewalt · Situationalistische Gewaltforschung · Entropie · Interaktionsordnung

\section{Violence and emotional energy}

Abstract This paper develops Randall Collins' theory of situationalistic violence in the central aspect of emotional energy. First, I supplement Collins' idea of positive emotional energy with the factor of negative emotional energy. Secondly, I model these two forms of energy as sets and relate them dynamically to each other. Thus, the entropy of emotional energy as a new factor in the development of violence plays a key role that has been neglected so far.

Keywords Violence $\cdot$ Situationalistic research of violence $\cdot$ Entropy $\cdot$ Interaction order 


\section{Einleitung}

Die aktuelle, viel diskutierte situationalistische Erforschung von Gewalt ist insbesondere darum bemüht, jene Mechanismen herauszuarbeiten, die zu physischer Gewalt führen (siehe insbesondere Collins 2008; Nassauer 2012, 2015a, b, 2016a, b; Equit et al. 2016). Vor allem Randall Collins schickt sich mit seinem Werk „Violence. A Micro-Sociological Theory“ aus dem Jahr 2008 an, den bisherigen dauerhaften „Ankündigungsmodus“ (Reemtsma 2006, S. 4) der soziologischen Gewaltforschung im Hinblick auf empirische Studien zu überwinden. Dafür geht er grundsätzlich davon aus, dass es „nicht um Gewalttäter, sondern um Gewaltsituationen“ (Collins 2008, S. 10) geht. Das Problem der Erklärung von Gewalt über sog. Hintergrundfaktoren wie Alter, Geschlecht, Status usw. ist nämlich, dass es immer mehr Personen mit denselben Merkmalen gibt, die keine Gewalt ausüben. Erklärungen von Gewalt qua Hintergrundfaktoren sind also nie hinreichend. ${ }^{1}$ Gewaltanwendung, so Collins, sei sowieso eher selten angesichts der zahlreichen konfliktuösen Situationen, d.h. in den allermeisten Fällen greifen Akteur*innen eben nicht zu Gewalt. Gewaltanwendung als Handlungsformat ist die Ausnahme in sozialen Interaktionen, und wenn es zu Gewalthandlungen kommt, dann werden diese mit auffälliger Inkompetenz durchgeführt. Folglich müsse die Erklärung von Gewalt vor allem die Frage klären, was notwendig ist, damit Akteur*innen in face-to-face-Interaktionen zumeist inkompetent Gewalt anwenden.

Was hindert Akteur*innen daran, häufiger Gewalt anzuwenden? Die bisherige Antwort von Collins (2008) fällt eindeutig aus: Die Überwindung der Konfrontationsanspannung und -angst ist notwendig für die Gewaltanwendung. Akteur*innen haben in vielen Interaktionen, insbesondere in Konfliktsituationen, Probleme, ,mit den engen dramaturgischen Beschränkungen einer Situation zurechtzukommen“ (Collins 2008, S. 546). Es kommt folglich zu einer Anspannung in der Interaktion, zu einer Gefährdung der für die Identität der beteiligten Akteur*innen höchstrelevanten Interaktionsordnung (Goffman 1994) ${ }^{2}$, was Collins (2008, 2009, 2016) mit den Begriffen der Konfrontationsanspannung und Konfrontationsangst als Formen emotionaler Energie umschreibt. Diese Zustände begründet Collins mit der anthropologischen Annahme des menschlichen Bedürfnisses nach (emotionaler) Stabilität in der Interaktion unter Anwesenden. Emotionale Energie ist dabei für ihn sowohl Antreiber als auch Resultat der Interaktionsdynamik ${ }^{3}$ : Die emotionale Energie hält die Akteur*innen an, in Interaktionen einzutreten, denn dort können sie ihr Zugehörigkeitsgefühl, Selbstbewusstsein, Enthusiasmus u.Ä. steigern. ${ }^{4}$ Soziale Rituale sorgen dafür, dass die Stabilisierung und Steigerung derartiger positiver emotionaler

\footnotetext{
${ }^{1}$ Zur Relevanz der Unterscheidung von notwendigen und hinreichenden Bedingungen für soziologische Erklärungen siehe Ragin (2000).

2 „Es war in all diesen Jahren mein Anliegen, Anerkennung dafür zu finden, dass diese Sphäre der unmittelbaren Interaktion der analytischen Untersuchung wert ist - eine Sphäre, die man, auf der Suche nach einem treffenden Namen, Interaktionsordnung nennen könnte" (Goffman 1994, S. 55).

${ }^{3}$ Bezweifelt werden kann allerdings, dass Emotionen alleine zur Erklärung von Strukturierungen genügen, etwa zur Erklärung der Dynamik von Märkten (siehe Sallach 2008).

${ }^{4}$ Collins (1993) geht an dieser Stelle von einer Art Maximierung emotionaler Energie aus. Ich werde zeigen, dass sich dies so generell als Aussage nicht halten lässt.
} 
Energie erwartungssicher möglich ist. Collins (2008, S. 247) bezeichnet die rituellen Interaktionsmechanismen als Emotionentransformator: „Die anfänglichen Gefühle werden [...] verstärkt und in emotionale Energie umgewandelt.“ Während Collins (2004) von Durkheim (1913) die Annahme der Genese von Solidarität aus kollektiver Erregung übernimmt, schließt er zugleich mit Goffman (1971), dass dies nicht nur für spezifische Phasen gesellschaftlichen Handelns gilt, sondern auch alltägliche Interaktionen als Interaktionsrituale zu analysieren sind (Greve 2012, S. 183) - und dies gilt auch für Gewaltsituationen bzw. für konfliktuöse Interaktionen. Es wird damit deutlich, dass emotionale Energie für Collins die zentrale Ressource innerhalb von Interaktionsdynamiken auch bei Gewalthandlungen darstellt. Denn als Konfrontationsanspannung bzw. Konfrontationsangst hemmt diese emotionale Energie zunächst die Ausübung von Gewalt, da die Menschen evolutionär ,,auf interaktives Mitgehen und Solidarität“ (Collins 2008, S. 46) gepolt seien. Wird diese emotionale Energie nicht in den positiven Bereich der Nähe einer „,kollektiven Efferveszenz“ (Durkheim 1913) überführt und verstärkt sich derart, dass die in Konfrontationsspannung- und angst liegende Hemmfunktion überwunden wird, dann kann es zu Gewalt kommen.

Der vorliegende Beitrag ${ }^{5}$ nimmt Collins an dieser Stelle bzgl. des theoretisch wichtigen Arguments der emotionalen Energie ernst. Dabei wird zugleich deutlich, dass sein Konzept der emotionalen Energie, trotz dessen zentraler Stellung innerhalb des Theoriegebäudes, einige Unschärfen aufweist (Rössel 1999, S. 30 f.) und offenkundig unterbestimmt ist (Cooney 2009; Greve 2012, S. 188 ff.). Laura Wolters (2019, S. $178 \mathrm{ff}$.) hat kürzlich die Gewaltforschung dafür kritisiert, diese Unbestimmtheit zwar zur Kenntnis zu nehmen, sich dennoch nicht weiter um die emotionssoziologischen Grundlagen von Collins' Gewaltheorie zu kümmern, sondern dessen Prämissen unreflektiert zu übernehmen. Unklar erscheint ihr insbesondere der Widerspruch, dass die ,für eine Erklärung des Ablaufs in Anspruch genommene Konfrontationsanspannung [...] gleichzeitig ein Hemmnis für Gewalt und als Ressource für Handlungsenergie auch noch deren Ermöglichungsbedingung “ ist (Wolters 2019, S. 191). Wolters (2019, S. 191) erhebt an dieser Stelle den Anspruch auf das Stellen grundsätzlicher Fragen, ,[d]a etwas aber nicht es selbst zugleich und sein Gegenteil sein kann“. ${ }^{6}$ Wir werden sehen, dass genau dies möglich ist, aber von Collins nicht modelliert wird. Collins an dieser Stelle ernst zu nehmen bedeutet also, das Konzept emotionaler Energie fortzuentwickeln, um diese theoretische Lücke zu schließen.

\footnotetext{
5 Ich danke Pascal Berger, Stefan Böschen, Andreas Braun, Edgar Hirschmann, Christina Laut, Bettina Mahlert, Hans-Jörg Sigwart, Lena Verneuer sowie den anonymen Gutachter*innen für ihre anregenden und hilfreichen Kommentare.

6 Dementsprechend schlägt sie eine andere „Emotionssoziologie der Gewalt“ (Wolters 2019, S. 199ff.) vor, die weitgehender ansetzt als der hier gemachte Vorschlag, da sie ein Konzept von Emotionalität präferiert, das jenseits der Dualismen von Emotion/Kognition, deskriptiv/normativ und Körper/Geist operiert. Damit würden einige zentrale Umbauten der Theorie von Collins notwendig, etwa ein neuer Situationsbegriff (Wolters 2019, S. 201), der Einschluss der Wirkung moralischer Wertungen (Wolters 2019, S. 202) bzw. leiblicher Erfahrungen in Bezug zu normativen Setzungen (Wolters 2019, S. 203). Der hier gemachte Vorschlag bleibt hingegen enger an Collins' theoretischen Arbeiten und bemüht sich um eine Erweiterung des Konzepts emotionaler Energie anstelle eines theoretischen Umbaus bzw. Austauschs mit derart weiterreichenden Konsequenzen.
} 
Einen wichtigen Fortschritt in der spezifischen Bestimmung von emotionaler Energie ist deshalb m.E. der Vorschlag von Boyns und Luery (2015), nicht nur positive, sondern auch negative emotionale Energie stärker zu berücksichtigen und in das Modell zu integrieren. ${ }^{7}$ Diesem Vorschlag möchte ich mich anschließen und weiter ausbauen. ${ }^{8}$ Dazu werde ich in einem ersten Schritt emotionale Energien als Mengen auslegen ${ }^{9}, \mathrm{zu}$ denen einzelne Ereignisse mehr oder weniger zugehörig sein können. Die daraus abgeleiteten Erkenntnisse, insbesondere der Faktor mengentheoretisch begründeter Entropie, erlauben als Maß für Interaktionsordnung zweitens eine Reformulierung der für Gewalthandlungen wichtigen Konfrontationsanspannung und Konfrontationsangst. Zuletzt werden dann einige Elemente aus Collins' Gewalttheorie spezifizierend neu gefasst.

\section{Emotionale Energien als Mengen}

Der erste Schritt der Neuerung von Collins' Modell besteht in der Einführung negativer emotionaler Energie. Während Collins positive emotionale Energie als Produkt gelungener Interaktionen versteht, ist negative emotionale Energie entsprechend das Ergebnis nicht gelungener Interaktionen. ${ }^{10}$ In ihrer positiven Ausprägung ist emotionale Energie eine Bewegung der Verbundenheit, in ihrer negativen Variante ist sie eine Dynamik der Feindseligkeit (Boyns und Luery 2015, S. 154). ${ }^{11}$ Beide Energien

\footnotetext{
${ }^{7}$ Es genügt nicht, Collins so zu deuten, als würde er negative emotionale Energie, definiert als Verlust positiver emotionaler Energie (Sallach 2008, S. 346f.), mitberücksichtigen. Zum einen hat Collins negative emotionale Energie nicht systematisch in seine Gewalttheorie implementiert und zum anderen sieht er sie nicht wie hier als eigenständige Energieform.

${ }^{8}$ Negative emotionale Energie ist als Idee nicht neu. Turner (2007, S. $195 \mathrm{ff}$.) beschreibt etwa die sozialstrukturellen Korrelate positiver wie negativer emotionaler Gestimmtheit und deren Zusammenspiel z. B. bei Dynamiken sozialen Wandels.

${ }^{9}$ In der mathematischen Re-Modellierung von Collins' Theorie werden weitere Schwierigkeiten offenkundig, die typisch für die verbalsprachliche Theoriebildung sind (McClelland 2014; vgl. Kron und Lasarczyk 2006): ,by constructing his theory primarily in verbal terms, Collins has relied for his definitions of key variables on a sociological tradition of theorizing that has emerged from commonsense discourse about social problems, and the resulting definitional ambiguity seriously limits the possibility of constructing more tightly defined computational models based on his theory. Models in the form presented by Collins simply do not provide suitable starting points for mathematical reformulation and computer simulation. Because the traditional approach of verbal definitions and schematic diagrams lacks the conceptual precision needed for modeling dynamic processes in terms of computer simulations, one must turn instead to some other form of theoretical modeling." (McClelland 2014, S. 104) Cooney (2009, S. $588 \mathrm{ff.}$.) identifiziert bei Collins vor allem im Abgleich mit der ,pure sociology“ von Donald Black (2004) Bestimmungsschwächen hinsichtlich der Relevanz von Hierarchien, dem Einfluss von Dritten sowie bei der Typologisierung von Gewaltformen.

${ }^{10}$ Nach Turner (2007, S. 201) steigt die Wahrscheinlichkeit negativer emotionaler Energien in Interaktionen, a) wenn die Teilnehmenden nicht dieselben, auf Emotionen bezogenen emotionale Phoneme und Syntax verwenden, b) wenn die Interaktion nicht in weitere korporative Einheiten oder nicht eindeutig in bestimmte institutionelle Domänen bzw. Stratifikationssysteme eingebettet sind, c) wenn die in die Interaktion eingebrachten kulturellen Symbole divergieren oder uneindeutig sind und d) wenn die Bedürfnisse der Beteiligten unklar, ambivalent und in der Begegnung nicht zu erfüllen sind.

${ }^{11}$ Schon daraus wird ersichtlich, dass Akteur*innen keine ausschließlichen Maximierer ihrer positiven Emotionen sind, sondern zugleich auch Reduzierer negativer Emotionen wie etwa Schmerz (siehe Scarry 1992).
} 
können zudem gesteigert (Zunahme) oder sie können verringert werden (Abnah$m e$ ). Wichtig sind die Kombinationen entgegengesetzter Wirkungen negativer wie positiver emotionaler Energien: So wird sowohl die Zunahme positiver emotionaler Energie als auch die Abnahme negativer emotionaler Energie in einer höheren Verbundenheit innerhalb der Interaktion resultieren, genauso wie eine Abnahme positiver und eine Zunahme negativer emotionaler Energie entzweiende Konsequenzen im Sinne der Feindseligkeit haben. Die Wirkungen der gleichgerichteten Zu- und Abnahme der Energien führt im Fall der beiderseitigen Abnahme von negativer und positiver emotionaler Energie in einen Zustand der Depression; im Falle der beiderseitigen Zunahme der emotionalen Energien in einen Zustand des Streits, hier mit Simmel (1992) verstanden als Vergesellschaftungsform, die eher Verbindendes als Konfliktform erreicht. ${ }^{12}$

Bei diesen kreuztabellierenden Kombinationen wird die emotionale energetische Aufladung nicht als dichotomer Entweder-Oder-Tatbestand begriffen, sondern jede Energie-Dimension im Anschluss an Bart Koskos (1995) „Fuzzy-Cube“ als eine Menge mit graduellen Zugehörigkeiten verstanden (siehe Abb. 1), die jeweils von voller bis zu keiner Zugehörigkeit reicht ([0/1] bzw. [1/0]), so dass der emotionale Zustand einen Raum aufspannt, welcher situativ volle emotionale Energie [1/1] bzw. keine emotionale Energie [0/0] beinhaltet.

Als Energie-Mengen betrachtet kann es zu Situationen kommen, die zugleich sowohl positiv als auch negativ emotional-energetisch aufgeladen sind. Emotionale Energien sind, so könnte man mit Beck (2004) formulieren, ein soziologischer Sachverhalt nicht exklusiver, sondern inklusiver Unterscheidungen. So kann bspw. eine grundsätzlich mit positiver emotionaler Energie aufgeladene Situation (z.B. das Zusammentreffen bei einer wissenschaftlichen Tagung) durch (nicht zu starke) negative emotionale Energie (z.B. durch kritische Diskussionsbeiträge) anregend stimuliert werden. Hier wird der Streit als wissenschaftliche Auseinandersetzung formiert. Negative emotionale Energie bewirkt reziprok u. U. zugleich eine gewisse Erhöhung positiver emotionale Energie, wenn die Abgrenzung nach außen die

\footnotetext{
${ }^{12}$ Streit wird nach Simmel aufgrund grundsätzlich entzweiender Antriebe wie Hass, Begierde, Neid usw. oft negativ bewertet, weil nicht zwischen Gesamt- und Individualnutzen unterschieden wird. Simmel hingegen analysiert Streit als eine Art umgekehrtes Kooperationsproblem: Es mag für die beteiligten Akteur*innen schlecht sein, muss es aber nicht für das Kollektiv: „Indem diese [Entzweiung] zwischen den einzelnen Elementen ihren verneinenden oder zerstörerischen Sinn entfaltet, wird daraus unbefangen geschlossen, dass sie in ebenderselben Weise auf das Gesamtverhältnis wirken müsse. In Wirklichkeit aber braucht, was zwischen Individuen, in bestimmter Richtung laufend und isoliert betrachtet, etwas Negatives, Abträgliches ist, innerhalb der Totalität der Beziehung keineswegs ebenso zu wirken." (Simmel 1992, S. 288, Herv. i.O.) Wenn ein Kampf nicht die Vernichtung des Anderen zum Ziel hat oder dieser nicht nur ausschließlich durch Kampflust motiviert ist, liegt nach Simmel immer ein verbindendes Element vor, das die Beteiligten selbst oft nicht erkennen können. Für die Einheitlichkeit miteinander verbundener Menschen fehlt „dem nachrechnenden Verstande [...] vielfach das Schema für diese Einheit“" (Simmel 1992, S. 292). Der Streit ist somit als Vergesellschaftungsform eine Synthese aus Verbindung und Trennung ich würde hier formulieren: eine Gemengelage aus positiver und negativer Energie - wie Simmel (1992, S. $318 \mathrm{ff}$.) etwa am Beispiel der Eifersucht ausführt: „Indem die Eifersucht den leidenschaftlichsten Hass an den gleichzeitigen Fortbestand der leidenschaftlichsten Liebe knüpfen kann, an das Nachwirken der innigsten Zusammengehörigkeit die Vernichtung beider Teile - [...] - ist die Eifersucht vielleicht diejenige soziologische Erscheinung, in der der Aufbau des Antagonismus über der Einheit seine subjektiv radikalste Gestaltung erreicht.“ (Simmel 1992, S. 322f., Herv. i.O.).
} 
Abb. 1 Energy-Cube

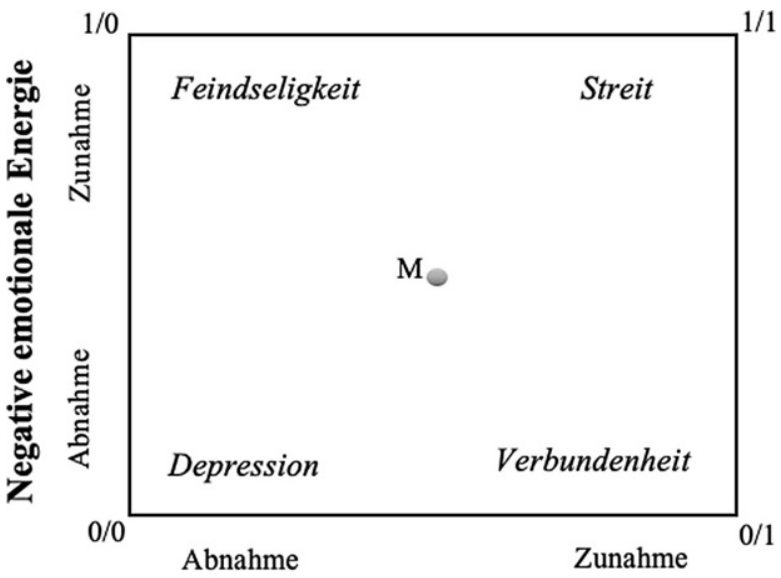

Positive emotionale Energie

Integration (Verbundenheit) nach Innen erhöht. Andersherum können Situationen, denen man grundsätzlich eine Steigerung oder Stabilisierung positiver emotionaler Energien zuschreiben kann (Treffen mit Freunden etc.), dann wesentlich zerstörerischer sein, wenn über ein bestimmtes Maß plötzlich die negative emotionale Energie zunimmt (z.B. durch Eifersucht unter den Freunden) und durch positive emotionale Energien ,begleitet“ wird, ohne dass die negativen emotionalen Energien abgeschwächt werden. Und zuletzt führt die Simultanität von hoher negativer Energie in einer Situation der Feindseligkeit und positiver emotionaler Energie eher zur Deeskalation als zu einem vielleicht sogar exzessiven Gewaltausbruch.

Emotionale Energien weisen nicht nur graduelle Zugehörigkeiten im Sinne inklusiver Unterscheidungen auf. Weiterhin verhalten sich positive und negative emotionale Energien innerhalb des Energieraums zueinander dynamisch, d.h. die Energien sind miteinander verbunden. Um nur ein Beispiel dafür zu nennen: Verliert ein/e Akteur*in z. B. die Verbundenheit zu einer Gruppe, dann verliert diese*r Akteur*in positive emotionale Energie und bildet infolgedessen möglicherweise Gefühle wie Scham oder Rache aus, d.h. er erfährt eventuell zugleich einen Zuwachs negativer Energie gegenüber ,seiner alten“ Gruppe. Das zeigt, der Energie-Level nimmt insgesamt nicht zwingend ab, nur weil mit dem Verlust der Verbundenheit die positive emotionale Energie verloren geht. Im Gegenteil kann die Energiebilanz insgesamt sogar höher sein, etwa wenn der/die Akteur*in sich vorher zwar nicht ,mit Haut und Haaren“ in der Interaktion (z. B. in seiner Partnerschaft) engagiert hat, ihn ein Ausschluss (Trennung) aber dennoch so sehr trifft, dass er nun „,voller Rache“ ist. Diese/r Akteur*in ist dann möglicherweise energetisch aufgeladener als zuvor, weil er nun mehr hasst als er davor geliebt hat.

Die unterschiedlichen graduellen Zugehörigkeiten und Dynamiken emotionaler Energien führen zu unterschiedlichen Erwartungs(un)sicherheiten über den Zustand der Ordnung der Interaktion. Diese Erwartungen über die Interaktionsordnung werde ich hier mengentheoretisch als Entropie der emotionalen Energien auffassen (vgl. 
Kosko 1995; Kron 2005, S. 205 ff.; Kron und Winter 2011). ${ }^{13}$ Je höher die Entropie ist, desto weniger geordnet erscheint den Akteur*innen die Interaktion. Oder anders formuliert: Je höher die Entropie der emotionalen Energien, desto geringer ist die Interaktionsordnung und desto weniger können die Akteur*innen erwarten, ihr Bedürfnis nach stabiler und sich steigernder positiver emotionaler Energie erfüllt zu bekommen. Die Entropie der emotionalen Energien fungiert hier als ein Maß der Interaktionsordnung und diese Entropie soll nach dem Wunsch der Akteur*innen, Collins folgend, möglichst gering sein. Genau deshalb greifen die Akteur*innen üblicherweise auf ein ganzes Set von nahezu rituellen Handlungen zurück, welche die Interaktionsordnung retten, d.h. die die Entropie so gering wie möglich halten. Man denke an interaktionsordnungsrettende Handlungen wie etwa ,rituelle Kundgaben, die einen Wechsel des Zugänglichkeitsgrades markieren“ (Goffman 1974, S. 119), oder an den „,korrektiven Austausch“: „Die Funktion der korrektiven Tätigkeit besteht darin, die Bedeutung zu ändern, die andernfalls einer Handlung zugesprochen werden könnte, mit dem Ziel, das, was als offensiv angesehen werden könnte, in etwas zu verwandeln, das als akzeptierbar angesehen werden kann." (Goffman 1974, S. 156).

Wichtig ist folglich das, was innerhalb des Energy-Cubes stattfinden kann: Ereignisse, die empirisch zu beiden Seiten der emotionalen Energien graduelle Zugehörigkeiten zwischen Null und Eins aufweisen und von denen Latour (1998) behauptet, dass diesem ,Zwischenbereich“ in der Gegenwartsgesellschaft besondere Aufmerksamkeit gewidmet werden müsse, weil sich dort alles abspiele: ${ }^{14}$ „In der Mitte, wo angeblich nichts passiert, ist fast alles.“ (Latour 1998, S. 160) Es mag z. B. Paare geben, bei denen die Unterscheidung zwischen Streit und Feindseligkeit nicht nur selbst vage ist (Codierungsvagheit, siehe Kron und Winter 2005, S. 379 ff.), sondern die ihre Verbundenheit gerade durch den Streit zu finden meinen, sodass graduelle Zugehörigkeiten zur Menge positiver Energien zu konstatieren sind (Zugehörigkeitsvagheit, siehe Kron und Winter 2005, S. 384 ff.). Dass selbst Feindseligkeiten Verbundenheit beinhalten können, hat Robert Axelrod (1988, S. 67 ff.) am Beispiel des Stellungskriegs vorgeführt. Und dass sowohl permanent vorhandene negative emotionale Energie qua Burnout oder Boreout (Dauerstress) als auch der komplette Mangel an emotionaler Energie in die Depression führen kann, ist ebenfalls bekannt. So kann das Paar, das immer wieder den Zyklus des durchaus feindselig ausgetragenen Streits mit anschließender Versöhnung durchläuft, zunehmend aufgrund der

\footnotetext{
13 Der Begriff der Entropie wird hier strikt im mathematisch-mengentheoretischen Sinn verwendet, nicht als physikalische oder informatische Größe. Es wäre zu überlegen (was hier nicht geleistet werden kann), ob der physikalisch-informatische Entropie-Begriff nicht weitere Zusammenhänge von Ordnung-Unordnung in Bezug auf Konfrontationen und Gewalt eröffnen könnte.

14 Ich lege viel Wert darauf, graduelle Zugehörigkeiten nicht als Ontologie misszuverstehen (vgl. Bauer 2018: 91). Das Modell sagt nicht, dass es Vagheiten bei emotionalen Energien ,gibt“! Sondern es lässt dem Beobachter offen, Vagheiten (im Sinne gradueller Zugehörigkeiten) zu beobachten und nicht gezwungen zu werden, Ereignisse dichotom (d.h. zweiwertig und mit den Gesetzen des Ausgeschlossenen Dritten sowie des Ausgeschlossenen Widerspruchs versehen) kategorisieren zu müssen. Entropie ist als ein zum exklusiven Unterscheiden (Beck 2004) alternatives Beobachterschema zu verstehen, und diese Alternative gilt sowohl für den wissenschaftlich oder sonst wie gesellschaftlich positionierten Beobachter (vgl. Kron 2014; Kron und Winter 2009). Zugleich ist Zweiwertigkeit als Möglichkeit mitberücksichtigt, dies sind die Mengen [0/1] und [1/0].
} 
durchgängig hohen Entropie in die Depression geraten. Die höchste Entropie ist der in dem Energy-Cube markierte Punkt M, der Mittelpunkt. An diesem Punkt herrscht die stärkste Interaktionsunordnung. Entropie, hier als Maß für Interaktionsordnung, bestimmt sich durch den Abstand eines Ereignisses zur nächst scharfen Menge positiver emotionaler Energie [0/1] im Verhältnis zur scharfen Menge der negativen emotionalen Energie [1/0] (vgl. Kosko 1995, S. 154). ${ }^{15}$ Da die Menge M denselben Abstand zu allen scharfen Mengen aufweist, ist sie graduell sowohl der vollen sowie der leeren Menge positiver emotionaler Energie zugehörig als auch der vollen sowie der leeren Menge negativer emotionaler Energie. Das bedeutet, ein Ereignis der Menge M ist gleichermaßen dem Streit, der Depression, der Feindseligkeit und der Verbundenheit zugehörig - der höchst ambivalenteste Zustand, der erreichbar ist und folglich die höchste Entropie aufweist.

Der anhand der kontextspezifischen Zu- bzw. Abnahme von emotionalen Energien gemessene Grad der Interaktionsordnung ist situativ natürlich hochgradig relevant, gerade wenn es mit Collins um Gewalt geht. Nahezu idealtypisch wird diese sensible Sollbruchstelle zwischen der Erwartung von Verbundenheit zur Steigerung positiver emotionaler Energie und der gleichzeitigen Angst um diese Verbundenheit in der modernen Gesellschaft wiederum anhand von Intimpartnerschaften figuriert (vgl. Collins 2008, S. 202 ff.): Zum einen sind Intimpartnerschaften jene Orte, an denen der/die einzelne Akteur*in noch damit rechnen kann - im Gegenteil zu allen anderen gesellschaftlichen Kontexten - in seiner Ganzheit wahr- und angenommen zu werden und nicht als Teil eines Rollen-Ensembles oder als systemspezifische Adresse (Fuchs 1997). Liebe (als symbolisch generalisiertes Kommunikationsmedium) ermöglicht die Unwahrscheinlichkeit der Kommunikation des Paares trotz der Formvorschrift, den/die Andere(n) komplett, mit allen Idiosynkrasien, zu berücksichtigen (Fuchs 1999). Dies ist legitimerweise erwartbar und drückt eine hochgradige vorgeordnete Interaktion - in einem: eine geringe Entropie - aus, denn die Erwartung der wechselseitigen Komplettberücksichtigung ist in das Programm romantischer Liebe eingeschrieben und institutionell, insbesondere im Rahmen der „Ehe“ mit Sanktionen bei Verstoß flankiert. Aus dieser besonderen erwartbaren und sogar einforderbaren Berücksichtigung der ganzen Individualität der Person erklärt sich die Bedeutsamkeit von Trennungen im Sinne höchster negativer emotionaler Energie, denn mit der Aufhebung der Intim-Gemeinschaft ist vorübergehend zugleich die Interaktionsordnung und damit die Einheit der individuellen Person sowie die Stabilität und die Möglichkeit der Steigerung positiver emotionaler Energie gefährdet - oder zumindest vorübergehend der gesellschaftliche Ort dafür genommen. Wenig überraschend erzeugt das Scheitern von Intimpartnerschaften ein hohes Maß an Gewalt als eine Form der Entropie-Reduktion als Resultat im zeitlich sich aufschaukelnden „Kampf um die Herbeiführung einer Übereinstimmung“ (Collins 2008, S. 203). Vor allem für Frauen, nach allgemeiner Vorstellung die physisch Schwächeren in einer hete-

\footnotetext{
15 Für den Fall, dass Vereinigungs- und Durchschnittsmenge maximal konvergieren, spricht Kosko vom „schwarzen Loch der Mengenlehre“, da nunmehr die Menge „A und nicht-A“ von der Menge „A oder nicht-A“ nicht länger unterschieden werden kann. Entropie meint die Unterschiedslosigkeit von Unterschieden, oder anders, der Grad der Unschärfe einer unscharfen Menge ist damit bestimmt durch die Ähnlichkeit (Untermengigkeitsgrad) zwischen einer Menge und ihrer Negation.
} 
rosexuellen Partnerschaft, liegt das höchste Risiko, Opfer von schwerer Gewalt bis hin zu Tötungsdelikten zu werden, in den Handlungen von ehemaligen männlichen Intimpartnern (Marneors 2008). In einer EU-weiten Erhebung zum Ausmaß der Gewalt gegen Frauen in Partnerschaften geben z. B. $22 \%$ der Befragten an, physische oder sexualisierte Gewalt erfahren zu haben (FRA 2014). Und laut der statistischen Auswertung des BKA (2018) zur partnerschaftlichen Gewalt für das Jahr 2017 wurden 16.692 Personen Opfer von gefährlicher, schwerer Körperverletzung oder einer solchen mit Todesfolge und 455 Personen wurden als Opfer von Mord und Totschlag registriert, in beiden Kategorien sind etwa $80 \%$ der Opfer weiblich. ${ }^{16}$

\section{Konfrontationsanspannung und Konfrontationsangst}

Die soziale Verrechnung von positiven und negativen emotionalen Energien ist also komplizierter als Collins' Darstellungen emotionaler Energie nahelegen. Wir können zum einen ergänzen: Während positive emotionale Energie bei den Akteur*innen den Wunsch nach ihrer Stabilisierung und Steigerung auslöst, erzeugt negative emotionale Energie eher ein Streben nach Reduktion der negativen Spannungen und einen Wandel hin zur positiven emotionalen Energie. ${ }^{17}$ Diese Spannungsreduktion kann durch eine ausgleichende Zufuhr positiver emotionaler Energien oder durch Ablenkungsstrategien etwa mittels des Konsums von Drogen, Sex, Sport oder Arbeit usw. versucht werden. Zum anderen soll die Entropie möglichst geringgehalten, d.h. die Interaktionsordnung auf möglichst hohem Niveau erhalten werden. Hohe Entropie geht mit einer geringen Interaktionsordnung einher, die Akteur*innen streben aber nach einer möglichst sicheren Interaktionsordnung. Entropie kann damit zugleich als $\mathrm{Maß}$ der Konfrontationsanspannung und damit der Interaktionsordnung fungieren: Je höher die Entropie, desto größer die Interaktionsunordnung, desto höher die Konfrontationsanspannung.

Weiterhin wichtig für die vorliegende Analyse ist, dass die Wahrscheinlichkeit der Manifestation einer feindseligen Interaktion mit der hohen Option von Gewalt umso größer ist, je energetischer die Interaktion insgesamt negativ emotional aufge-

\footnotetext{
16 Collins interessiert sich auch hier vor allem für die Art der Verstrickung der Paare in ihren Mikrodynamiken. Die im Laufe einer Paarbeziehung entstehenden mikrodynamischen Muster können mit sozialstrukturellen Faktoren korrelieren, etwa wenn ein Mann eher zu Dominanz in der Paarbeziehung neigt, je größer die Statusdifferenz zur Ehefrau ist. Collins (2008, S. 232) legt dennoch Wert darauf, dass es die Mikroverstrickungen inkl. des gemeinsamen Umgangs des Paares mit Konfrontationsanspannung und Konfrontationsangst sind, die Vorkommen, Art und Ausmaß der Gewalthandlungen bestimmen. Und dies bedeutet im Umkehrschluss, dass Paare solche „situationsgerechten Techniken“ (Collins 2008, S. 232) erlernen können, die deeskalierend wirken.

17 Nach Turner (2007) erhöhen negative emotionale Energien die Wahrscheinlichkeit der Anwendung ,,defensiver Strategien und Mechanismen“ bei den Akteur*innen. Wie oben erwähnt, können negative Energien aber auch stimulierend wirken, sofern sie grundsätzlich in einem Kontext positiver Energien auftreten und ein bestimmtes Maß nicht überschreiten. Mit Bezug auf das oben verwendete Beispiel der wissenschaftlichen Tagung formuliert: Eine gewisse kurzfristige Erhöhung der Entropie durch die wissenschaftliche Auseinandersetzung kann unter der Gewissheit, dass es nicht zum körperlichen Kampf kommen wird, den weiteren kritischen Diskurs anregen. Dieser selbst ist Teil der Interaktionsordnung, erhöht und senkt die Entropie also in einem.
} 
Abb. 2 Violence-Cube

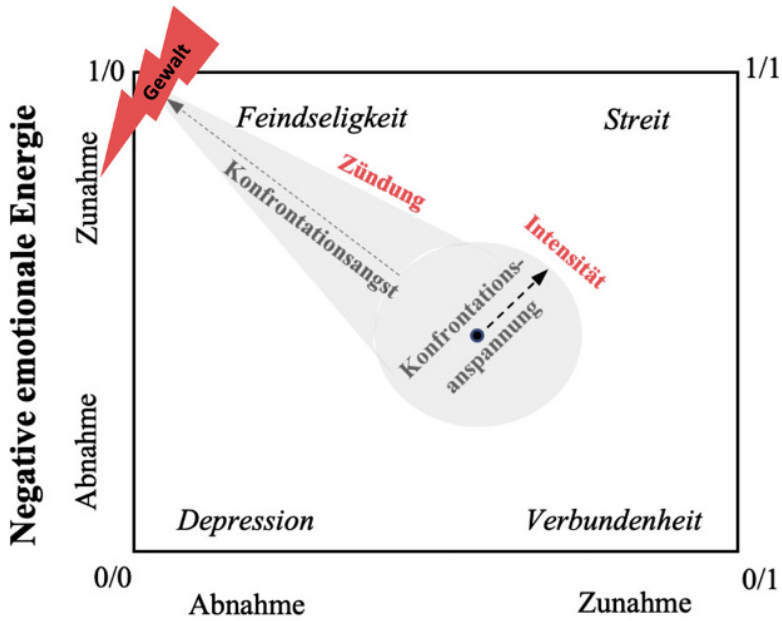

Positive emotionale Energie

laden ist. Bei Gewalt stehen positive und negative emotionale Energien nicht gleich wirkend nebeneinander. Der negativen emotionalen Energie kommt eine besondere Bedeutung zu, da alleine ihre Zunahme Gewalt wahrscheinlicher macht. Diese Wahrscheinlichkeit wird zudem weiter erhöht durch die entgegengesetzte Energiedynamik der Zunahme negativer bei gleichzeitiger Abnahme positiver emotionaler Energie. Die bei Collins wenig von der Konfrontationsanspannung differenzierte Konfrontationsangst besteht nun darin, dass die Akteur*innen die gleichzeitige Zunahme negativer bei Abnahme positiver emotionaler Energie fürchten. Denn das bedeutet, dass sich eine Interaktionsordnung einstellt, welche von negativer emotionaler Energie bestimmt ist. Ein Zustand der Feindseligkeit, der - wie etwa ein Krieg (Rotte 2019) hochgradig geordnet, dennoch zugleich sehr unangenehm und vor allem nicht einer Zunahme positiver emotionaler Energie dienlich ist. Gewalt, das deutet sich hier an, ist ein Mittel, diesen unangenehmen Zustand der Feindseligkeit zu beenden. Gewalt ist nämlich nicht nur ganz grundsätzlich eine Form der Entropie-Reduktion, sondern Gewalt ist ebenfalls die Möglichkeit der Auflösung negativer emotionaler Energie in einer feindseligen Situation mit geringer Entropie, die kaum mehr Aussicht auf eine Zunahme positiver emotionaler Energie (wenigstens in Richtung Streit) erlaubt. Wenn die Positivität nicht mehr im Horizont der Möglichkeiten erscheint, bleibt die Auflösung der Negativität. Dies ist die Doppelfunktion von Gewalt: Sie kann Entropie auflösen und eine Unordnung der Interaktion in eine (Gewalt-)Ordnung überführen. Dies ist, wie wir sehen werden, eher selten. Die zweite Funktion ist, dass Gewalt die Angst vor zu hoher negativer und zu geringer positiver emotionaler Energie kompensiert, indem sie dieser Situation ein vorauseilendes Ende setzt. An diesem Punkt angekommen, scheint insbesondere eine vermeintlich offenkundige Schwäche beim Gegenüber den Ausschlag zum Gewaltakt zu liefern (Collins 2008, S. 202 ff.; Nassauer 2015a, 2016b). Das Gewalthandeln bestätigt dann das, was man zuvor befürchtet hat (die Bestimmtheit der Situation durch pure negative emotionale Energie) und beendet es zugleich sehr schnell, wenn die Gewalt erfolgreich gewesen 
ist. Ein Schlag genügt. Und danach ist die Situation eine andere - das war das Ziel des Gewalthandelns.

Konfrontationsangst, verstanden als Furcht vor einer situativen Bestimmtheit durch ausschließlich negative emotionale Energie, zündet damit den Gewaltausbruch. D.h., die Konfrontationsangst scheint die „Zündung“ eines gewalttätigen Konflikts zu erklären (siehe Abb. 2), während die Konfrontationsanspannung eher für die Intensität (und damit für das Ausmaß der Destruktivität) von Feindseligkeiten relevant zu sein scheint (Boyns und Luery 2015, S. 163).

\section{Folgerungen: Vorwärtspanik, Seltenheit, Deeskalation, Polarisierung}

Mit diesem mengentheoretischen Modell positiver und negativer emotionaler Energien können nun verschiedene Ableitungen von Collins' Gewalttheorie präziser reformuliert werden. Ich möchte diese Präzisierungen hier im Hinblick auf die sog. „Vorwärtspanik“, die Seltenheit von Gewalt, die Deeskalation von Gewalt durch Langeweile und die Polarisierung vornehmen.

\subsection{Vorwärtspanik und negative Energie}

Die mikrosoziologische Gewaltforschung ist sich einig, dass es verschiedene Wege in den „Tunnel der Gewalt“ gibt. Der Begriff des Tunnels (Collins 2008, S. $544 \mathrm{ff}$.) signalisiert, dass es - einmal in diesen hineingeraten - nur noch wenige Gleise gibt, diesen zu durchfahren. Und ein Gleis führt direkt zur Gewalt. Einmal auf dieses Gleis gelangt, führt kein Weg mehr aus der Gewalt hinaus, solange man in diesem Tunnel steckt. Die sogenannte Vorwärtspanik - das Aufstauen und Entladen von emotionaler Energie auf Schwächere - symbolisiert als extremste Form den Weg in und durch den Tunnel der Gewalt: „Der Typus der Vorwärtspanik, die asymmetrische Verstrickung der im Augenblick Dominierenden und der passiven Verlierer, ist die spektakulärste Version, der tiefste Punkt im Tunnel der Gewalt.“ (Collins 2008, S. 544; siehe Collins 2015) Vorwärtspanik bedeutet für Collins, dass die Konfrontationsanspannung in der Interaktion bereits sehr hoch ist, einer der beteiligten Akteur*innen eine Schwäche beim Anderen ausmacht und daraufhin seine emotionale Energie in Form eines gewalttätigen Angriffs auf den Anderen umlenkt. Wir können nun ergänzen: Die Konfrontationsanspannung macht die Intensität der gewaltvollen Vorwärtspanik plausibel, die Konfrontationsangst erklärt, wieso die Interaktion eine solche explosive Dynamik annehmen kann. ${ }^{18}$ Es ist die Entladung der negativen emo-

\footnotetext{
18 Es muss hier offenbleiben, wie Vorwärtspanik etwa mit der Größe der Angst oder mit der Geschwindigkeit der Interaktionsdynamik zusammenhängt. Zur Relevanz von Zeit siehe auch Hoebel (2014).
} 
tionalen Energie ${ }^{19}$ in einen (aufgrund der zuvor aufgebauten Konfrontationsanspannung) heftigen gewaltsamen Angriff, der jegliche sachlich-rationale Angemessenheit des Handelns vermissen lässt (Collins 1993), so dass Beobachter*innen regelmäßig einen „Gewaltexzess“ attestieren. Es wird dann auf den Unterlegenen eingeschlagen und eingetreten, obwohl dieser bereits kampfunfähig am Boden liegt. ${ }^{20}$ Wenn man tief und lange genug in diesem Tunnel der Gewalt verbracht hat, gewöhnt man sich erst langsam wieder an die Helligkeit nach dem Tunnel, d.h. es benötigt eine gewisse Zeit, bis sich die negative emotionale Energie entladen hat und ggf. durch eine hinreichend positive emotionale Energie ausgeglichen wurde. Das von dem/der Gewalt ausübenden Akteur*in gewünschte Ergebnis - die Auflösung hoher negativer emotionaler Energie - ist durch die Gewalt und zugleich spätestens mit der Beendigung der Gewalt am Ende des Tunnels erreicht.

\subsection{Negative Energie und die Seltenheit von Gewalt}

Weiterhin wird nun auch klarer, weshalb es trotz zahlreicher konfliktuöser Interaktionen empirisch so selten zur Gewaltanwendung kommt. Collins (2008, S. $118 \mathrm{ff.}$, 2009, S. $568 \mathrm{ff}$.) geht davon aus, dass der Grund darin läge, dass Akteur*innen den Verlust positiver emotionaler Energie scheuen und diese Furcht sogar größer sei als jene vor der möglichen Verletzung des eigenen Körpers. Diese Annahme erscheint nicht besonders plausibel. Cooney (2009, S. 591 ff..) verweist darauf, dass Gewalt, wie auch viele andere Formen abweichenden Verhaltens (z.B. Verkauf von Betäubungsmitteln, Betrug, Geschwindigkeitsübertretungen), graduelle Phänomene darstellen, bei denen ,kleinere“ Abweichungen naturgemäß öfter vorkommen als „,schwere“ Vergehen. „Naturgemäß“ bedeutet hier, dass das Verhältnis von der Schwere der Abweichung zur Häufigkeit einer sog. „Power-Law-Verteilung“ entspricht (siehe Buchanan 2000 für Kriege, Clauset et al. 2010; Kron (2015) für Terrorismus; Kron und Grund 2009 für gesellschaftstheoretische Überlegungen). Intensität und Häufigkeit stehen in einem umgekehrten Verhältnis zueinander und das „Power-Law“ beschreibt die spezifische Art und Weise, in der die Häufigkeit des Auftretens eines Ereignisses mit der Stärke des Ereignisses zusammenhängt.

\footnotetext{
19 Mit Mazur (2009) kann man negative emotionale Energie hier auch als hohen Cortisol-Spiegel spezifizieren, welcher in einer Situation eigener hoher Dominanz (d.h. in Kombination mit einem hohen Testosterongehalt) zum Gewaltexzess führt: ,,forward panic“ is explained by changes in cortisol and testosterone that occur during battle. When individual contests within the killing zone are decided, the testosterone of each winner rises, increasing his assertiveness, while the testosterone of each loser falls, lessening his will to compete. In the winner, no longer threatened, cortisol falls and he is uninhibited from pressing his attack. In the terror stricken loser, cortisol goes higher, promoting his flight or, if that option is unavailable, causing incapacitation. Hormonally fortified assertive- ness, or lack of it, entrains combatants on either flank. The losing soldiers, under the combined effects of lowered testosterone and raised cortisol, turn from defiance to abject submission, while winners ride over them." (Mazur 2009, S. 445).

20 Bspw. wurde der später für seine Zivilcourage posthum geehrte Dominik Brunner bei einer Auseinandersetzung mit zwei Jugendlichen am 12.09.2009 am S-Bahnhof Solln (München) innerhalb von einer Minute 22 Mal geschlagen und getreten. Er verstarb letztlich aufgrund eines schwachen Herzens, welches dieser Stresssituation nicht standhielt.

21 „The general message of a power law is indeed intuitively obvious; the precise mathematical relationship, however, is not at all inevitable." (Ball 2004, S. 243).
} 
Die genaue mathematische Beziehung muss hier nicht interessieren ${ }^{21}$, es ist offenkundig, dass weniger gewaltvolle Abweichungen häufiger vorkommen als extrem gewaltvolle Handlungen. Die Seltenheit von Gewalt ist so gesehen nichts anderes als die situative Manifestation eines abstrakteren Makro-Zusammenhangs, der möglicherweise mikro-soziologisch nicht ausschließlich durch die situative Angst vor dem Verlust positiver Energie erklärt werden kann.

Plausibler scheint es folglich, mit Goffman (1971, S. 192) die Sorge um die Unversehrtheit des eigenen Körpers einzubeziehen und als dauerhafte Anpassungstechnik zu verstehen: „Eine gewisse Sorgfalt muss immer angewandt werden. Sorge tragen ist ein ständiger Zustand des Seins." Der Grund für die relative Seltenheit von Gewalt kann folglich mit dem hier vorgestellten Modell in der dargestellten Konfrontationsangst gesehen werden: Konfrontationsangst geht mit der Aussicht auf die Auflösung hochgradig negativer emotionaler Energien durch Gewalt einher, die die Sorge um die Unversehrtheit des eigenen Körpers beinhaltet. Dies hält die Akteur*innen von der Gewaltanwendung oft lange ab und lässt sie auf jene menschlichen Reaktionsweisen zurückgreifen, die in konfliktuösen Situationen auch bei starkem Stress noch abrufbar sind: Flucht, Verstecken, Dominanz- oder Unterwerfungsverhalten (Miller 2011). Für dieses Argument spricht auch die hormonelle Re-Interpretation dieser Vorgänge (Mazur 2009), sofern das „Stress-Hormon“ Cortisol in konfliktuösen Situationen ansteigt und in Aussicht steht, dass dieser Stress zunächst gut z. B. mit Flucht als Handlungsalternative abgebaut werden kann: „Cortisol is released into the bloodstream when people are stressed [...]. It mobilizes the body for either ,fight or flight"." (Mazur 2009, S. 445) Gerade wenn das Gegenüber stark genug zum Widerhandeln scheint, ist das Stress- bzw. Cortisol-Niveau derart hoch, dass die Ausübung von Gewalt gegen diese unmöglich erscheint.

Zudem muss für die Selektion von Gewalt als Handlung eine individuell maximal hohe negative emotionale Energie vorhanden und zugleich eine situative Gelegenheit gegeben sein, die vorhandene negative emotionale Energie in Richtung einer Überwindung der Konfrontationsangst durch Gewalt umzulenken. Konfrontationsangst bedeutet hier: Angst vor der zerstörerischen Kraft der eigenen negativen emotionalen Energie sowie vor denselben möglichen Energien des Gegners in dieser Situation. Eingepreist ist damit die Angst vor einer eigenen Verletzung bzw. vor Verletzung des Anderen. Ist die Summe dieser Ängste hoch genug und gibt es keine situative Gelegenheit, die eigene negative emotionale Energie in Gewalt einzulenken, dann stehen dem/der Akteur*in statt der Entscheidung zur Gewalthandlung alternativ eben auch die genannten Handlungsalternativen, mindestens der Fluchtversuch, zur Verfügung.

Collins' Idee, dass Gewalt selten ist, weil die Akteur*innen eher um Reduktion der Konfrontationsanspannung bemüht seien, kann ebenfalls in dem hier vorgestellten Modell reformuliert werden. Aus meiner Sicht erklärt das Auftreten positiver emotionaler Energie trotz bestehender Feindseligkeiten zwischen den Akteur*innen - also noch vor etwaigen Gewalthandlungen -, weshalb ein Konflikt insgesamt abschwächt (Boyns und Luery 2015, S. 162f.): Das Einbringen positiver emotionaler Energie in eine feindselige Situation erhöht nämlich die Entropie. Und die mit der erhöhten Entropie verbundene Vagheit verringert wiederum die „Zündung“, zunächst auf Kosten erhöhter Konfrontationsanspannung, weil durch die Zunahme positiver emotionaler Energie eine Wiederherstellung der Interaktionsordnung letzt- 
lich im Sinne der Verbundenheit in Aussicht gestellt ist, diese aber im Zustand der Feindseligkeit noch sehr unsicher erscheint. Selbst wenn die negative emotionale Energie zunächst hoch bleiben sollte, kann sich die Entropie durch Zunahme positiver emotionaler Energie erhöhen, also z. B. in Richtung eines Streits. Vielleicht mag es dann für den unbedarften Außenstehenden so erscheinen, als stünde ein Gewaltausbruch unmittelbar bevor, etwa weil die Protagonisten einen ,monkey dance“ (Miller 2008, S. 42f., 2011, S. 26 ff., 2015, S. 24; Miller und Kane 2012, S. 15 ff.) vorführen und sich Brust an Brust stehend beschimpfen und bedrohen. Der Begriff des ,Tanzes“ zeigt an, dass dies als ein Dominanz- bzw. Territorial-Ritual verstanden werden kann, welches eine eigene Interaktionsordnung der Bedrohung generiert, die im Normalfall nicht in Gewalt übergeht.

Konfrontationsanspannung an sich erklärt so gesehen weniger die Seltenheit von Gewalt, als vielmehr die Häufigkeit des Vorkommens negativer emotionaler Energie, also die Häufigkeit von Interaktionen, in denen Gewalt aus den genannten Gründen überhaupt als Handlungsalternative in den Blick gerät. Auf der einen Seite bemühen sich Akteur*innen zwar um die Vermeidung der Verletzung der Interaktionsordnung (um die Vermeidung hoher Entropie) zur Rettung positiver emotionaler Energie, auf der anderen Seite, so Collins (2008, S. 555), führen die vielen möglichen „Brüche im Mikrointeraktionsfluss [...] Menschen an die Schwelle der Gewalt.“ Das bedeutet, die Menschen werden im everyday life regelmäßig mit negativer emotionaler Energie versorgt. Die Relevanz gelingender Interaktionsordnung ist so hoch, dass Akteur*innen diese nicht verletzen wollen und zugleich aufgrund dieser hohen Relevanz schon bei Kleinigkeiten empfindlich reagieren, wenn eine solche Verletzung droht. Neurologisch gesehen kann die mit der Verletzung der Interaktionsordnung einhergehende negative emotionale Energie ein Ausmaß erreichen, welches einer existenziellen Bedrohung gleichkommt, gespiegelt in der neurologischen Aktivität des monkey brains (Miller 2015, S. $8 \mathrm{ff}$.), das dem/der menschlichen Akteur*in als Säugetier vorgaukelt, das Leben wäre durch die disäquilibrierte Verbundenheit zu dem Anderen in der Interaktion tatsächlich bedroht. ${ }^{22}$ Mit Mühlmann (2005) könnte man sagen, dass Bedrohungen der Verbundenheit maximalen Stress - Todesangst suggerieren. Todesangst ist zweifelsohne eine starke negative emotionale Energie! Die Zunahme negativer emotionaler Energie ist dann das Resultat der „Konflikte über den Gesprächsablauf auf der Mikroebene“ (Collins 2008, S. 545). Dazu mag es genügen, dass die Beteiligten gegen den allgemein gültigen „Takt“ verstoßen

\footnotetext{
22 Monkey Brain meint jenen Teil des menschlichen Gehirns, der mit dem limbischen System verknüpft und auf unser soziales Verhalten bezogen ist. Für die praktische Gewaltforschung (z. B. auch van Horne und Riley 2014) wichtig ist, dass ,,the monkey brain cannot distinguish between humiliation and death. For much of our evolution, being cast out of the tribe was to be sentenced to a slow and lonely death. The monkey shows this and fears being ostracized above all things." (Miller 2015, S. 9) Todesangst entsteht folglich nicht nur dann, wenn der Mensch in Todesgefahr ist (wofür der Hirnstamm, das „Reptiliengehirn“, zuständig ist), sondern auch wenn er sich sozial gefährdet fühlt - etwa wenn die Interaktionsordnung gefährdet scheint. Das bedeutet, der Mensch wird manchmal gewalttätig, weil er um seine soziale Verbundenheit (z. B. um seinen Status, um seine Wertschätzung, um seinen Rang innerhalb der Hierarchie seiner Gruppe etc.) fürchtet. Zu evolutionären Bedingungen von Gewalt siehe auch Meyer (2004).
} 
(siehe Simmel 2011; vgl. Goffman 1969, 1971) ${ }^{23}$, sich unterbrochen haben, sich ins Wort gefallen sind, sich beleidigt haben usw. Kommt es zu keinem positiv energetischen Ausgleich oder zum Abbruch der Situation, steigt die negative emotionale Energie und damit die Konfrontationsangst an. Erst in der Nähe eines maximalenergetischen Niveaus negativer Emotionalität - resultierend aus höchster Konfrontationsangst als Folge hoher Konfrontationsanspannung - wird Gewalt als alternative Handlungsoption statt Flucht, Verstecken, Dominanz- oder Unterwerfungsverhalten gewählt. Collins selbst hat ein Gefühl für diesen Zusammenhang von Konfrontationsanspannung und Konfrontationsangst, kann diesen aber zugleich nicht systematisch in sein Modell theoretisch einbetten: „Diese Mikroeskalation erwächst oft unmittelbar aus dem Bemühen, sich Gehör zu verschaffen und den anderen umzustimmen. Die Auseinandersetzung darum, wer das Wort hat, wird immer lauter, die Atmosphäre immer angespannter. Beim Versuch, sich durchzusetzen und den Anderen zum Zuhören zu bewegen, spannen sich die Muskeln an, und irgendwann entlädt sich diese Anspannung in einem Faustschlag." (Collins 2008, S. 547) Der mit diesen Vorgängen verbundene Stress ist für die allermeisten Akteur*innen derart hoch, dass die Gewalthandlung selbst dann in weit überwiegendem Maße völlig inkompetent und irrational in dem Sinne ausgeführt wird, dass unter den vielen Optionen der Gewalthandlungen nicht die effektivsten oder effizientesten Handlungen selegiert werden.

\subsection{Deeskalation durch Langeweile und negative Energie}

Hier wird weiterhin deutlich, weshalb Collins als Form der Intervention im Konfliktfall auf Langeweile setzt ${ }^{24}$ : „This is practical advice from micro-sociology: you keep a confrontation from escalating by keeping it at the level of stalled repetitions, until it de-escalates quite literally from boredom." (Collins 2012, S. 12f.) Collins' Idee ist, dass beide Seiten sich in ihren Gesten und vor allem in ihrer emotionalen Intensität spiegeln und dies so lange in einer Art Gleichgewicht tun, dass keine Seite emotionale Dominanz ausüben und diese in eine gewaltvolle Überwindung der Konfrontationsangst übersetzen, also in Collins' Perspektive keinen emotionalen Vorteil aus der Situation ziehen kann. In der wechselseitigen Wahrnehmung der Konfrontationsangst liegt zwar zum einen das Risiko, dass der Andere in der eigenen Angst eine Schwäche erkennt und vorwärtspanisch agiert, zum anderen beinhaltet sie den-

\footnotetext{
23 „Darum ist in der Gesellschaft das Taktgefühl von so besonderer Bedeutung, weil dies die Selbstregulierung des Individuums in seinem persönlichen Verhältnis zu anderen leitet, wo keine äußeren oder unmittelbar egoistischen Interessen die Regulative übernehmen.“ (Simmel 2011, S. $180 \mathrm{f}$.).

${ }^{24}$ Collins (2012, S. $11 \mathrm{ff}$.) gibt immer wieder Hinweise auf Chancen der Deeskalation eines Konflikts, die sich aus seiner situationalistischen Perspektive ergeben. Interessanterweise sind seine Vorschläge wohl wenig geeignet für die Präventionsberatung, obwohl ihm diese noch vor der soziologischen Erklärung am Herzen liegt: „Der Einwand ist sicher richtig, zu sagen, dass die situationalistische Perspektive eine große Erklärungslücke hinterlässt, da sie sich vor allem auf das auslösende Moment der Gewalt konzentriert. [...] Ein [...] Grund für die diese Forschungsrichtung ist, dass ich situative Techniken für deutlich vielversprechender halte, was die Vermeidung von Gewalt angeht." (Collins 2019, S. 63). Wenn Collins andeutet, dass die Eskalation eines Konflikts dadurch verhindert wird, dass Menschen manchmal vermeiden, sich am Konflikt zu beteiligen, dann bleibt offen, wie ich Menschen präventiv dazu bringe, sich aus einem Konflikt rauszuhalten.
} 
noch die Chance, die Gewalt nicht zu schnell zu zünden. Da es schwierig ist, hohe negative emotionale Energie aufrechtzuhalten, wenn diese keine zusätzliche situative Zufuhr erhält und beide Akteur*innen, wie gesagt, eher darum bemüht sind, negative emotionale Energie zu reduzieren und ggf. stattdessen positive emotionale Energie zu erzeugen, sind diese im Konfliktfall auch eher bereit, die Eskalation bzw. den Konflikt abzubrechen und diesen nicht in Gewalthandlungen zu überführen. Das Nicht-Zünden der Gewalt ist in diesem Sinne ,langweilig“ und für beide Akteur*innen ein Hinweis auf eine Chance der Reduktion negativer emotionaler Energie schlicht durch Abbruch der Feindseligkeit, was an sich die Situation zugleich energetisch bereits positiv emotional auflädt, etwa wenn beide Protagonisten gemeinsam die Situation abbrechen, indem sie z. B. simultan auseinandergehen.

\subsection{Polarisierung und negative Energie}

Zu guter Letzt kann nun auch die Rolle von Polarisierung neu bestimmt werden. ${ }^{25}$ „Polarization is the great enemy“, so Collins (2012, S. 18). Den Grund für die gewalteskalierende, oft mit Gräueltaten einhergehende Wirkung vor allem ideologischer Polarisierung erläutert Collins (2012) anhand von Überlegungen zur Konfliktdynamik, die er im Rahmen seiner Theorie der Interaktionsrituale reformuliert und spezifiziert hat. Dabei geht Collins zunächst davon aus, dass die Dynamik gewalttätiger Interaktionen ein Resultat verschiedener Abweichungsverstärkungen im Sinne positiver Rückkopplungen (vgl. Schimank 2000, S. 207 ff.) sind ${ }^{26}$, die letztlich dazu führen, dass Gruppensolidarität und Konflikt sich wechselwirkend verstärken. Nach Collins (1993, S. 206, 2004, 2012, S. 2 ff.) sind für Interaktionsrituale generell vier Bedingungen wichtig, welche ebenfalls wesentlichen Einfluss auf Konfliktdynamiken haben: Erstens muss eine Interaktion unter mindestens zwei Anwesenden (,physical density“) mit zweitens einer nach innen und außen bestimmbaren Grenze (,boundedness") gegeben sein. Konflikte haben hier die Funktion, den Zusammenhalt nach innen zu erhöhen und dadurch den Konflikt nach außen zu verstärken. Drittes muss es einen gemeinsamen Aufmerksamkeitsfokus der Gruppe (, focus of attention") geben, auf den sich das Zusammenhandeln richtet. Ein solcher Aufmerksamkeitsfokus ist durch feindliche Aktionen anderer Gruppen sowie durch den Blick auf die eigenen Akteur*innen gegeben (Kämpfer, Opfer), was wiederum die Konflikthaftigkeit verstärkt. Und viertens ist eine gemeinsam geteilte emotionale Stimmung (,commodity of emotional good“) notwendig. Diese emotionale Stimmung kann auch durch Angst vor dem Feind oder durch Wut auf den Gegner entstehen,

\footnotetext{
25 Diese Neubestimmung von Polarisierung ist notwendig, weil Collins seltsamerweise bei seinen Analysen in diesem Beitrag (2012) stets von rivalisierenden Gruppen mit mehreren Mitgliedern auszugehen scheint und nicht von der Möglichkeit reiner face-to-face-Situationen, in denen sich ausschließlich zwei Akteur*innen gegenüberstehen. Ob für die akute Situation „Mann gegen Mann“ die Abnahme von Gruppensolidarität und ideologischer Polarisierung relevante Anker für Präventionsmaßahmen sind, darf bezweifelt werden, denn hier spielen die von ihm (2012, S. 13f.) hervorgehobenen zeitlichen Dynamiken von Gruppensolidarität genauso wenig eine Rolle wie seine logistischen Überlegungen zum Ressourceneinsatz über die Zeit oder zum Wegfall von Unterstützern.

26 McClelland (2014, S. 104) kritisiert Collins' Konzentration auf Abweichungsverstärkungen und die Vernachlässigung von Abweichungsdämpfungen (,negative feedback loops“).
} 
was noch einmal den Konflikt verstärkt. Die Folgen erfolgreicher Interaktionsrituale sind erstens eine Erhöhung der Gruppensolidarität, aus der der Einzelne die Bereitschaft zieht, sich für die Gruppe ggf. zu opfern. Zweitens folgt die Produktion idealisierter Mitgliedschaftssymbole, welche die Identifikation mit dem verstärken, was in der Gruppe als Gut und Böse gilt. Und drittens wird nach Collins eine hohe emotionale, positive Energie erzeugt, konkretisiert als Selbstvertrauen und Begeisterung bezüglich des Konflikts im Sinne eines unbedingten Glaubens an den Willen zum Sieg.

Polarisierung als intervenierender Faktor verschärft nun den Konflikt, weil dichotome Anschauungen es den Akteur*innen erstens erleichtern, sowohl sich selbst als eindeutig gut und die Anderen als vollkommen böse zu kennzeichnen. Zweitens hilft die Polarisierung, auch heftigste Gewalttaten als moralisch „reinigende“ Handlungen (Douglas 1985) im Sinne der Gruppensymbolik zu deuten.

Im Ergebnis ist Collins voll und ganz an dieser Stelle zuzustimmen (siehe auch De Crescenzo 1997, S. 152f.; Kron und Winter 2014). Zugleich können wir mittels des hier präsentierten Modells die Wirkung von ideologischer Polarisierung spezifizieren. Eine polarisierende Einstellung bewirkt eine Radikalisierung bei der Selbstevaluierung bezüglich emotionaler Energie, d.h. jede Vagheit des selbstgefühlten Zustands wird unmittelbar auf ein eindeutiges Befinden hin ausgedeutet. Jedes Gefühl bedeutet dann, dass die negative bzw. positive emotionale Energie entweder da ist oder nicht da ist, respektive die Ordnung der Interaktion ist entweder vorhanden oder sie ist nicht vorhanden - tertium non datur. Ereignisse bzw. Gefühlszustände mit graduellen Zugehörigkeiten zu verschieden Mengen werden durch Polarisierung direkt vereindeutigt (Bauer 2018) und die Vagheit geklärt. Der Vorteil dieses Vorgehens ist, dass man sich nicht mehr mit der Entropie beschäftigen muss. Der Nachteil ist, dass man zum einen sehr viel schneller in den Bereich der Feindseligkeit und damit zur Gewalt kommt. Das Entweder-Oder zündet die Gewalt sehr viel schneller als das Sowohl-als-Auch. Zum anderen und damit einhergehend ist Entropie eben nicht nur Fluch, sondern beinhaltet die Chance der Ausdeutung gerade aufgrund der damit verbundenen Vagheit: Sofern eine Interaktion nicht eindeutig feindselig ist [1/0], enthält sie eben auch (Spuren-)Elemente von Verbundenheit. Jede nicht-eindeutige Feindseligkeit ist Menge sowohl einer feindseligen als auch einer mit Verbundenheit geprägten Interaktion, wenn auch zu unterschiedlichem Grade. Und dies ermöglicht den Akteur*innen, eine Deeskalation der Feindseligkeiten entlang der Zunahme der positiven emotionalen Energie bzw. der damit verbundenen immerhin vorhandenen Aussicht auf Verbundenheit zu versuchen, was im Sinne doppeltkontigenten Ordnungsstrebens gelingen kann (siehe Luhmann 1984, S. 148 ff.). 


\section{Schlussbemerkung}

Situationen der Gewalt sind energetisch voraussetzungsreich. ${ }^{27}$ Damit Gewalt geschieht, müssen Akteur*innen hinreichend viel negative emotionale Energie entwickelt haben und dürfen kaum eine Chance sehen, die Interaktion in eine Richtung zu entwickeln, die möglichst geringe Entropie bei Zunahme positiver emotionaler Energie erlaubt. Praktische Präventions- und Interventionsprogramme setzen genau an diesen Schaltstellen an (vgl. Staller et al. 2018): Man vermittelt den Akteur*innen bspw. Selbstregulationskompetenzen, damit sie in Situationen hoher Entropie oder negativ-emotionaler Energie noch alternative Handlungsstrategien zu Gewalt kognitiv in den Blick nehmen können. Oder man zeigt ihnen, wie sie die kommunikative Anschlussfähigkeit in Richtung positiver emotionaler Energie erhöhen, etwa durch „gewaltfreie Kommunikation“ (Rosenberg 2001). Oder, um ein letztes Beispiel zu nennen, man vermittelt solche Selbstschutzkompetenzen zum Umgang mit dem Gewalthandeln des Gegenübers, welche geeignet sind, den Gewaltvorgang zu unterbrechen und die Wahrscheinlichkeit erhöhen, dass sich die negative emotionale Energie abbauen kann (,law enforcement“, siehe Körner und Staller 2018).

Greve (2012, S. 189f.) kritisiert zurecht, dass Collins das Management (positiver wie negativer) emotionaler Energien nicht ebenfalls im Anschluss an Goffmans (1967) Arbeiten zu Techniken der Imagepflege ausgearbeitet hat, z.B. die Vermeidung von Verlust positiver bzw. des Zuwachses negativer emotionaler Energie durch „Vermeidungsrituale“ oder durch die genannten Selbstregulierungs-, Kommunikations- oder Selbstschutzkompetenzen. Sofern man nämlich das Bemühen um emotionale Stabilität im Sinne eines Emotionsmanagements als Teil der individuellen Identität betrachten möchte, kann man Collins' Konzept durchaus als Spezifikation des Akteurs/der Akteurin als Goffmensch (Hitzler 1991, 1992, 1994) verstehen, der existenziell bedroht ist, wenn er seine Identität nicht angemessen behaupten bzw. seine positiven emotionalen Energien nicht stabilisieren und steigern sowie seine negativen emotionalen Energien nicht verarbeiten bzw. diese vermeiden kann, wofür die Interaktionsordnung eine höchstrelevante Bedingung darstellt. Goffman wiederum hat dargelegt, wie Akteur*innen schon im normalen Alltag permanent darum bemüht sind, ihre Identitäten zu behaupten, indem sie Interaktionen so gestalten, dass aktuale und virtuale soziale Identität möglichst wenige Diskrepanzen aufweisen, z.B. durch Techniken des Umgangs mit Stigmata (Goffman 1967) oder durch aktive Rollenausgestaltung (Goffman 1973) etc. Die Rituale des everyday life sind folglich extrem wichtig, weil sie Erwartungssicherheit über die Interaktionsprozesse generieren und darüber die individuelle Identität - wir ergänzen: und als dessen Teil ebenfalls das Management emotionaler Energie - sichern (Collins 2004; Goffman 1971). Alltagsrituale dienen auf diese Weise der Prävention von Gewalt. Die soziologische Beobachtung der Veränderungen von Alltagsritualen erfüllt somit eine

\footnotetext{
27 Gewalt gilt in der Moderne als geeignetes Mittel zur Herstellung gesellschaftlicher Ordnung im Abweichungsfalle, nicht aber für Interaktionsordnungen. Deshalb liegt als normative Forderung ,die Gewalt im Staate“ und darf von Akteur*innen in Interaktionen trotz anthropologisch gegebener „Aktionsmacht“ (Popitz 1986) nur dann eingesetzt werden, wenn es im Sinne von „Notwehr“ dem unmittelbaren eigenen Schutz (bzw. dem Dritten) dient.
} 
wichtige Aufgabe für die Gewaltprävention, insbesondere wenn es gelänge darzulegen, unter welchen Bedingungen Situationen positive und wann sie eher negative Energien auslösen. Auch hier kann lediglich weiterer Forschungsbedarf angemeldet werden. Als abschließende These würde ich mit Goffman solchen Situationen eine Erzeugung positiver Energien unterstellen, die es vermögen, dass alle beteiligten Akteur*innen ihre in dieser Situation gegebenen Bedürfnisse hinreichend befriedigen können. Rituale sind so gesehen bewährte und festgelegte Strategien zur Befriedigung von Bedürfnissen der Ritual-Beteiligten - mindestens das Bedürfnis nach Interaktionsordnung (geringer Entropie) wird damit erfüllt. Zugleich ist dies nicht alles: Ein ritualisiertes Essen unter Freunden z. B. mag zudem das Bedürfnis nach Verbundenheit, Spaß, Anerkennung (z. B. für das zubereitete Essen), Austausch usw. nähren.

Negative Energien dürften folglich eher solche Situationen zeitigen, welche die Bedürfniserfüllung verhindern oder Bedürfnisse gar in Mangel setzen. Sofern man den Schulbesuch bspw. als ein kollektives Ritual beschreiben möchte, erstaunt doch, wie häufig Schüler*innen dieses Ritual energetisch negativ spiegeln. Im Rahmen der hier vorgebrachten These wäre z. B. zu vermuten, dass die Institution Schule mit ihren Bewertungsritualen dem Bedürfnis nach Anerkennung entsprechen kann. Im Rahmen der schulischen „Anerkennungssphäre“ (Honneth 1994) ist dieses Bedürfnis zugleich häufig ausschließlich durch Leistung und Gehorsam zu erfüllen, so dass Schule aufgrund dieser Strategiewahl die Erfüllung anderer Bedürfnisse wie Autonomie, Wertschätzung oder auch Schlaf zumindest gefährdet. Dass Gewalt an Schulen im durkheimschen Sinne normal ist (siehe Durkheim 1976), wundert folglich wenig, weil Schulen offenbar aufgrund dieser Strategiewahl negative Energien auslösen können. Das Noten- bzw. Versetzungssystem etwa versetzt die Schüler*innen per se in einen nahezu dauerhaften Unsicherheitszustand. ${ }^{28}$ Gewalthandlungen sind dann wiederum Alternativstrategien zu Gehorsam und Leistung zur Erfüllung z.B. von Anerkennung (in der peer-group) und zugleich zur Absorption von Unsicherheit. Es kommt vermutlich nicht zu noch mehr Gewalt, weil Schulen ebenfalls (schon durch ihre hohe Erwartungssicherheit der zeitlich, sachlichen und sozialen Abläufe) ebenfalls positive Energien auslösen. Das Einüben bedürfniserfüllender Rituale, so könnte man die These fortspinnen, fördert und fordert solche Kompetenzen der Akteur*innen, welche mindestens der Unfähigkeit entgegenwirken, mit der Nähe der Anderen umzugehen. Dass schulische und andere Alltagsrituale diese Kompetenzen zunehmend nicht fördern, sondern eher auf ein Gegenteil hinwirken, ist ein Zustand, den Zygmunt Bauman als Adiaphorisierung auch und gerade für die neoliberal-flüchtige Gegenwart diagnostiziert hat (Bauman 2000a, b; siehe Kron 2020). Eine Diagnose, die relevant ist für Konflikt- bzw. Gewaltsituationen (Kron 2019).

Funding Open Access funding provided by Projekt DEAL.

\footnotetext{
28 Bestandssicherheit kann als Teil einer erwartungssicheren (entropiearmen) Interaktionsordnung verstanden werden. Wissen Schüler*innen nicht, ob sie versetzt werden, sind sowohl zumindest (schulische) Anerkennung als auch Bestandssicherheit massiv gefährdet.
} 
Open Access Dieser Artikel wird unter der Creative Commons Namensnennung 4.0 International Lizenz veröffentlicht, welche die Nutzung, Vervielfältigung, Bearbeitung, Verbreitung und Wiedergabe in jeglichem Medium und Format erlaubt, sofern Sie den/die ursprünglichen Autor(en) und die Quelle ordnungsgemäß nennen, einen Link zur Creative Commons Lizenz beifügen und angeben, ob Änderungen vorgenommen wurden.

Die in diesem Artikel enthaltenen Bilder und sonstiges Drittmaterial unterliegen ebenfalls der genannten Creative Commons Lizenz, sofern sich aus der Abbildungslegende nichts anderes ergibt. Sofern das betreffende Material nicht unter der genannten Creative Commons Lizenz steht und die betreffende Handlung nicht nach gesetzlichen Vorschriften erlaubt ist, ist für die oben aufgeführten Weiterverwendungen des Materials die Einwilligung des jeweiligen Rechteinhabers einzuholen.

Weitere Details zur Lizenz entnehmen Sie bitte der Lizenzinformation auf http://creativecommons.org/ licenses/by/4.0/deed.de.

\section{Literatur}

Axelrod, Robert. 1988. Die Evolution der Kooperation. München: Oldenbourg.

Ball, Philip. 2004. Critical Mass. How One Thing Leads to Another. New York: Farrar, Straus and Giroux. Bauer, Thomas. 2018. Die Vereindeutigung der Welt. Über den Verlust an Mehrdeutigkeit und Vielfalt. Stuttgart: Reclam.

Bauman, Zygmunt. 2000a. Ethics of individuals. In Individualisierung und soziologische Theorie, Hrsg. Thomas Kron, 203-217. Opladen: Leske + Budrich.

Bauman, Zygmunt. 2000b. Liquid modernity. Cambridge: Polity Press.

Beck, Ulrich. 2004. Der kosmopolitische Blick oder: Krieg ist Frieden. Frankfurt a.M.: Suhrkamp.

Black, Donald. 2004. Violent structures. In Violence: from theory to research, Hrsg. Margaret A. Zahn, Henry H. Brownstein, und Shelly L. Jackson, 145-158. Cincinnati: Anderson.

Boyns, David und Sarah Luery. 2015. Negative Emotional Energy: A Theory of the "Dark-Side" of Interaction Ritual Chains. Social Sciences 4(1):148-170.

Buchanan, Mark. 2000. Ubiquity. New York: Bloomsbury.

Bundeskriminalamt. 2018. Partnerschaftsgewalt. Kriminalistische Auswertung 2017. Wiesbaden: BKA.

Clauset, Aaron, Lindsay Heger, Maxwell Young, und Kristian Skrede Gleditsch. 2010. The strategic calculus of terrorism: substitution and competition in the Israel-Palestine conflict. Cooperation and Conflict 45:6-33.

Collins, Randall. 1993. Emotional energy as the common denominator of rational action. Rationality and Society 5:203-230.

Collins, Randall. 2004. Interactional ritual chains. Princeton: Princeton University Press.

Collins, Randall. 2008. Dynamik der Gewalt. Eine mikrosoziologische Theorie. Hamburg: Hamburger Edition.

Collins, Randall. 2009. The micro-sociology of violence. British Journal of Sociology 60:566-576.

Collins, Randall. 2012. C-escalation and D-escalation: a theory of the time-dynamics of conflict. American Sociological Review 77:1-20.

Collins, Randall. 2015. Vorwärtspaniken und die Dynamik von Massengewalt. In Gewaltmassen. Über Eigendynamik und Selbstorganisation kollektiver Gewalt, Hrsg. Axel T. Paul, Benjamin Schwalb, 204-230. Hamburg: Hamburger Edition.

Collins, Randall. 2016. Einfahrten und Ausfahrten des Tunnels der Gewalt. In Situationen der Gewalt, Hrsg. Claudia Equit, Axel Groenemeyer, und Holger Schmidt, 14-39. Weinheim: Beltz Juventa.

Collins, Randall. 2019. An der Schwelle zur Gewalt. Ein Gespräch mit Randall Collins. Mittelweg 36 28:60-69.

Cooney, Mark. 2009. The scientific significance of Collins's violence. The British Journal of Sociology 60:586-594.

de Crescenzo, Luciano. 1997. Die Kunst der Unordnung. München: Knaus.

Douglas, Mary. 1985. Reinheit und Gefährdung. Berlin: Reimer.

Durkheim, Emile. 1913. Die elementaren Formen des religiösen Lebens. Frankfurt a.M.: Suhrkamp.

Durkheim, Emile. 1976. Die Regeln der soziologischen Methode. Frankfurt a.M.: Suhrkamp.

Equit, Claudia, Axel Groenemeyer, und Holger Schmidt (Hrsg.). 2016. Situationen der Gewalt. Weinheim: Beltz Juventa.

FRA - Agentur der Europäischen Union für Grundrechte. 2014. Gewalt gegen Frauen: eine EU-weite Erhebung. Luxemburg: Amt für Veröffentlichungen. 
Fuchs, Peter. 1997. Adressabilität als Grundbegriff der soziologischen Systemtheorie. Soziale Systeme 3:57-79.

Fuchs, Peter. 1999. Liebe, Sex und solche Sachen. Zur Konstruktion moderner Intimsysteme. Konstanz: UVK.

Goffman, Erving. 1967. Stigma. Über Techniken der Bewältigung beschädigter Identität. Frankfurt a.M.: Suhrkamp.

Goffman, Erving. 1969. Wir alle spielen Theater. Die Selbstdarstellung im Alltag. München: Piper.

Goffman, Erving. 1971. Interaktionsrituale. Über Verhalten in direkter Kommunikation. Frankfurt a.M.: Suhrkamp.

Goffman, Erving. 1973. Interaktion: Spaß am Spiel. Rollendistanz. München: Piper.

Goffman, Erving. 1974. Das Individuum im öffentlichen Austausch. Mikrostudien zur öffentlichen Ördnung. Frankfurt a. M.: Suhrkamp.

Goffman, Erving. 1994. Die Interaktionsordnung. In Interaktion und Geschlecht, Hrsg. Hubert Knoblauch, 50-104. Frankfurt a. M.: Campus.

Greve, Jens. 2012. Emotionen, Handlungen und Ordnungen: Überlegungen zu Randall Collins. In Emotionen, Sozialstruktur und Moderne, Hrsg. Annette Schnabel und Rainer Schützeichel, 181-199. Wiesbaden: VS.

Hitzler, Ronald. 1991. Goffmans Perspektive. Notitzen zum dramatologischen Ansatz. Sozialwissenschaftliche Informationen 20:276-281.

Hitzler, Ronald. 1992. Der Goffmensch. Überlegungen zu einer dramatologischen Anthropologie. Soziale Welt 43:449-461.

Hitzler, Ronald. 1994. Kleine Strategien. Zur alltäglichen „Protopolitik des Selbst“. Ästhetik und Kommunikation 23:83-86.

Hoebel, Thomas. 2014. Organisierte Plötzlichkeit. Eine prozesssoziologische Erklärung antisymmetrischer Gewaltsituationen. Zeitschrift für Soziologie 43:441-457.

Honneth, Axel. 1994. Kampf um Anerkennung. Zur moralischen Grammatik sozialer Konflikte. Frankfurt a.M.: Suhrkamp.

van Horne, Patrick, und Jason Riley. 2014. Left of bang. New York: Steven Pressfield.

Körner, Swen, und Mario S. Staller. 2018. From system to pedagogy-towards a non-linear pedagogy of self-defense in the law enforcement and the civilian domain. Security Journal 31:645-659.

Kosko, Bart. 1995. Fuzzy logisch. Eine neue Art des Denkens. Düsseldorf: Econ.

Kron, Thomas. 2005. Der komplizierte Akteur. Vorschlag für einen integralen akteurtheoretischen Bezugsrahmen. Münster: LIT.

Kron, Thomas. 2014. Autopoiesis und Hybride - zur Formkatastrophe der Gegenwartsgesellschaft. Zeitschrift für Theoretische Soziologie, 2014(2):220-252.

Kron, Thomas. 2015. Reflexiver Terrorismus. Weilerswist: Velbrück.

Kron, Thomas. 2019. Zur Prävention individualisierter Gewalt. In Soziologie für den Öffentlichen Dienst. Konflikte und Gewalt in öffentlichen Organisationen, Hrsg. Johanna Groß, 266-277. Hamburg: Maximilian.

Kron, Thomas. 2020. Moral und Gewalt - Wie viel Moral ist in Gewaltsituationen möglich? Ein Blick aus der Perspektive Zygmunt Baumans. In Fehlfarben der Postmoderne. Weiter-Denken mit Zygmunt Bauman, Hrsg. Kristin Platt. Weilerswist: Velbrück. im Erscheinen.

Kron, Thomas, und Thomas Grund. 2009. Society as a selforganized critical system. Cybernetics and Human Knowing 16:65-82.

Kron, Thomas, und Christian W.G. Lasarczyk. 2006. Zur sozionischen Notwendigkeit mechanistisch-soziologischer Erklärungen. In Reflexive soziale Mechanismen. Von soziologischen Erklärungen zu sozionischen Modellen, Hrsg. Marco Schmitt, Michael Florian, und Frank Hillebrandt, 105-137. Wiesbaden: VS.

Kron, Thomas, und Lars Winter. 2005. Fuzzy Systems - Überlegungen zur Vagheit sozialer Systeme. Soziale Systeme 11:370-394.

Kron, Thomas, und Lars Winter. 2009. Fuzzy thinking in sociology. In Views on fuzzy sets and systems from different perspectives. Philosophy and logic, criticisms and applications, Hrsg. Rudi Seising, 301-320. Berlin: Springer.

Kron, Thomas, und Lars Winter. 2011. Die radikale Unbestimmtheit des Sozialen. In Uneindeutigkeit als Herausforderung. Risikokalkulation, Amtliche Statistik und die Modellierung des Sozialen, Hrsg. Daniel Fischer, Wolfgang Bonß, Thomas Augustin, Felix Bader, Michaela Pichllbauer, und Dominikus Vog1, 187-215. Neubiberg: Universität der Bundeswehr München.

Kron, Thomas, und Lars Winter. 2014. Entscheidungshandeln und Fuzzy-Logik. In Handbuch Modellbildung und Simulation in den Sozialwissenschaften, Hrsg. Norman Braun, Nicole J. Saam, 363-396. Wiesbaden: Springer VS. 
Latour, Bruno. 1998. Wir sind nie modern gewesen. Versuch einer symmetrischen Anthropologie. Frankfurt a. M.: Suhrkamp.

Luhmann, Niklas. 1984. Soziale Systeme. Grundriß einer allgemeinen Theorie. Frankfurt a.M.: Suhrkamp.

Mazur, Allan. 2009. A hormonal interpretation of Collins's micro-sociological theory of violence. Journal for the Theory of Social Behaviour 39:434-447.

Marneros, Andreas. 2008. Intimizid - Die Tötung des Intimpartners. Stuttgart: Schattenauer.

McClelland, Kent. 2014. Cycles of conflict: a computational modeling alternative to Collins's theory of conflict escalation. Sociological Theory 32:100-127.

Meyer, Peter. 2004. Grundlagen menschlicher Gewaltbereitschaft. Beiträge evolutionärer Forschung. In Gewalt, Hrsg. Wilhelm Heitmeyer, Hans-Georg Soeffner, 383-410. Frankfurt a.M.: Suhrkamp.

Miller, Rory. 2008. Meditations on violence. Wolfeboro: YMAA.

Miller, Rory. 2011. Facing violence. Preparing for the unexpected. Wolfeboro: YMAA.

Miller, Rory. 2015. Conflict communication: a new paradigm in conscious communication. Wolfeboro: YMAA.

Miller, Rory, und Lawrence A. Kane. 2012. Scaling force. Dynamic decision-making under threat of violence. Wolfeboro: YMAA.

Mühlmann, Heiner. 2005. MSC Maximal Stress Cooperation: Die Antriebskraft der Kulturen. Berlin: Springer.

Nassauer, Anne. 2012. Violence in demonstrations: a comparative analysis of situational interaction dynamics at social movement protests. Dissertation. Berlin: Humboldt-Universität.

Nassauer, Anne. 2015a. Effective crowd policing: empirical insights on avoiding violence. Policing: An International Journal of Police Strategies \& Management 38:3-23.

Nassauer, Anne. 2015b. Developing a micro-situational theory of crowd violence. In Cultures, social bonds and the dynamics of violence, Hrsg. Elizabeth Crespo Kebler, 39-53. Oxfordshire: Inter-Disciplinary Press.

Nassauer, Anne. 2016a. Theoretische Überlegungen zur Entstehung von Gewalt in Protesten: Eine situative mechanismische Erklärung. Berliner Journal für Soziologie 25:491-518.

Nassauer, Anne. 2016b. From peaceful marches to violent clashes: a micro-situational analysis. Social Movement Studies 15:1-16.

Popitz, Heinrich. 1986. Phänomene der Macht. Tübingen: J.C.B. Mohr.

Ragin, Charles C. 2000. Fuzzy-set social science. Chicago: University of Chicago Press.

Reemtsma, Jan P., 2006: Die Natur der Gewalt als Problem der Soziologie. Mittelweg 36 15(5):2-25.

Rössel, Jörg. 1999. Konflikttheorie und Interaktionsrituale. Randall Collins' Mikrofundierung der Konflikttheorie. Zeitschrift für Soziologie 28:23-43.

Rosenberg, Marshall B. 2001. Gewaltfreie Kommunikation. Eine Sprache des Lebens. Paderborn: Junfermann.

Rotte, Ralph. 2019. Das Phänomen Krieg: Eine sozialwissenschaftliche Bestandsaufnahme. Wiesbaden: Springer.

Sallach, David L. 2008. Modeling emotional dynamics. Currency versus field. Rationality and Society 20:343-365.

Scarry, Elaine. 1992. Der Körper im Schmerz. Die Chiffren der Verletzlichkeit und die Erfindung der Kultur. Frankfurt a.M.: Fischer.

Schimank, Uwe. 2000. Handeln und Strukturen. Weinheim: Juventus.

Simmel, Georg. 1992. Soziologie. Untersuchungen über die Formen der Vergesellschaftung. Frankfurt a.M.: Suhrkamp.

Simmel, Georg. 2011. Soziologie der Geselligkeit. In Aufsätze und Abhandlungen (1909-1918), $177-183$. Frankfurt a.M.: Suhrkamp.

Staller, Mario, Andy Abraham, Jamie M. Poolton, und Swen Körner. 2018. Avoidance, de-escalation and attacking. An expert coach consensus in self-defense-practice. Movement: Journal of Physical Education and Sports Science 11:213-214.

Turner, Jonathan H. 2007. Human emotions. A sociological theory. London: Routledge.

Wolters, Laura. 2019. Vorwärtspanik am Wickeltisch? Zum Verhältnis von Emotionen und Gewalt. Mittelweg 36 28:177-203.

Thomas Kron 1992-1997 Studium der Soziologie, Politik- und Medienwissenschaften an der HeinrichHeine-Universität Düsseldorf, 2000 Promotion zum Dr. rer.pol an der Otto-Friedrich-Universität Bamberg, 2005 Habilitation an der FernUniversität in Hagen. Seit 2007 Univ.-Professor für Soziologie an der RWTH Aachen. Forschungsschwerpunkte sind Soziologische Theorien (insbesondere System- und Akteurtheorien), Fuzzy-Logik in der Soziologie und die Gewaltforschung. 